\title{
Comparison between Lambert and Maxwell Approaches in the Modelling of Microwave Heating of Liquid Foods
}

\author{
Rino Apicella, Vittorio Romano \\ Department of Industrial Engineering, University of Salerno, Fisciano, Italy \\ Email: rapicella@unisa.it,vromano@unisa.it
}

How to cite this paper: Apicella, R. and Romano, V. (2019) Comparison between Lambert and Maxwell Approaches in the Modelling of Microwave Heating of Liquid Foods. Engineering, 11, 1-13.

https://doi.org/10.4236/eng.2019.111001

Received: October 24, 2018

Accepted: January 6, 2019

Published: January 9, 2019

Copyright (อ 2019 by author(s) and Scientific Research Publishing Inc. This work is licensed under the Creative Commons Attribution International License (CC BY 4.0).

http://creativecommons.org/licenses/by/4.0/

Open Access

\begin{abstract}
Microwave heating of liquid foods in laminar flow through a circular tube has been modeled. In particular, skim milk as a Newtonian fluid and apple sauce and tomato sauce as non-Newtonian fluids have been considered. The temperature profiles have been obtained solving the motion and energy equations in transient regime and Maxwell's equations in the frequency domain. $\mathrm{Nu}$ merical resolution of Finite Element Method has been implemented in Comsol Multiphysics. The generation term due to the microwave heating has been evaluated according both to Lambert's law and Poynting theorem. Finally, a comparison between the two methods has been made in order to check to what extent the results obtained with the simpler Lambert's law approximation are comparable with those deriving from the exact solution of Maxwell equations. Dielectric properties are considered to be temperature dependent.
\end{abstract}

\section{Keywords}

Continuous Microwave Heating, Lambert's Law, Maxwell Equations, Poynting Theorem, Non-Newtonian Fluids, FEM

\section{Introduction}

Microwave heating has been utilized since the 1940s [1] in different fields such as polymer and ceramics industries [2] [3] and medicine [4] [5]. However, food processing is the largest consumer of microwave energy, that can be employed for cooking, thawing, tempering, drying, freeze drying, pasteurization, sterilization, baking, heating and re-heating. In microwave heating, electromagnetic field polarizes the molecules of dielectric materials and creates dipole moments that cause these molecules to rotate. The resulting molecular friction causes heat 
generation in the body. Due to intrinsic heat generation capability, microwave heating can provide prompt rise of temperature within the low thermal conductive products, especially in food items. On the other hand, the presence of hot and cold spots determines an uneven heating. This is primarily caused by the non-uniform distribution of microwave energy in the foodstuff, due to factors such as dielectric loss, penetration depth, thickness, shape and size of the sample.

Microwave heating of solid foods has been largely investigated in the last years, writing the energy equation as a conductive heat transfer with a generation term.

The latter has been modeled by many authors, using two different approaches to evaluate the effects of the microwave distribution: by solving the Maxwell's equations [2] [6] [7] or by applying the Lambert's law [8] [9]. The Lambert's law is a simple power formulation that was believed to simulate temperature profiles for Cartesian geometries and for cylindrical geometries with high radius. Ayappa et al. [2] deduced the minimum value of the characteristic sample dimension to successfully apply the law for slabs; Oliveira and Franca [7] found that this value was higher for cylinders than for slabs. Finally, Romano et al. [10] correctly applied Lambert's law considering the geometry of the sample, thus finding the same field of application for both the shapes and a power concentration along the central axis for a cylindrical domain, according with the experimental observations [9].

As regards fluids, there are fewer studies and the most are about batch processes, conducted only by means of Maxwell equations solution [11] [12].

As regards continuous microwave heating, the problem of temperature distribution analysis in liquid foods that flow in cylindrical ducts was faced by Romano and Apicella [13], taking into account also the velocity field and different rheological characteristics. In that work, generation term due to microwaves in the energy balance was modeled with Lambert's law while in the present work, problem is faced also by solving the electromagnetic problem and results are then compared to check at what extent Lambert's law estimation produces valid and accurate outcomes respect to Maxwell equation solution.

Sheryl Barringer and others [14] compared Lambert's law and Maxwell equations solution on agar gel made slabs, observing that only exact solution of Maxwell equation can appreciate electric field oscillations; moreover, Lambert's law seems to underestimate the absorbed power and, therefore, the temperature profile. However, an approximately exponential decay from surface towards the inner of sample is followed in both cases.

In the present paper, an analysis on liquid foods and a cylindrical geometry is made. The results of the two different models hypothesis are compared in terms of absorbed power and temperature profile.

\section{Mathematical Model}

\subsection{Physical System}

The physical system to be modeled is a cylindrical horizontal tube with a length $L=0.3 \mathrm{~m}$ and a radius $R=0.02 \mathrm{~m}$ (Figure 1 ) in which different liquid foods are 
heated by microwave irradiance in the radial direction. A laminar flow is realized by a pressure drop between the inlet and the outlet sections. Only the axial component of the velocity is different from zero and it is a function of the radial direction : $v_{z}=v_{z}(r)$. The temperature, even though the microwave penetration is only radial, is also function of the axial direction by the effect of the flow field: $T=T(r, z)$.

The aim is to check the temperature profile and to compare results obtained with Lambert's law and Maxwell equations resolution.

\subsection{Balance Equations}

The mathematical model consists of the following three differential equations in vector form with their boundary conditions [15].

$$
\begin{gathered}
\frac{D}{D t} \rho=-\rho(\underline{\nabla} \cdot \underline{v}) \quad \text { (continuity equation) } \\
\rho \frac{D}{D t} \underline{v}=-\underline{\nabla p}-[\underline{\nabla} \cdot \underline{\tau}]+\rho \underline{g} \quad \text { (momentum equation) } \\
\rho c_{p} \frac{D}{D t} T=k \nabla^{2} T+Q \quad \text { (energy equation) }
\end{gathered}
$$

In cylindrical coordinate system, Equations (1) and (2) for the specific problem become:

$$
\begin{gathered}
\frac{\partial v_{z}}{\partial z}=0 \\
\rho \frac{\partial v_{z}}{\partial t}=-\frac{\partial}{\partial r}\left(r \tau_{r z}\right)+\frac{P_{\text {in }}-P_{\text {out }}}{L}
\end{gathered}
$$

with the following initial and boundary conditions.

As initial condition, the fluid is stationary.

$$
@ t=0, v_{z}=0, \forall r, z
$$

As boundary conditions, we assume no slipping at the wall

$$
\text { @ } r=R, v_{z}=0, \forall z, \forall t>0
$$

And we consider the symmetry on the axis.

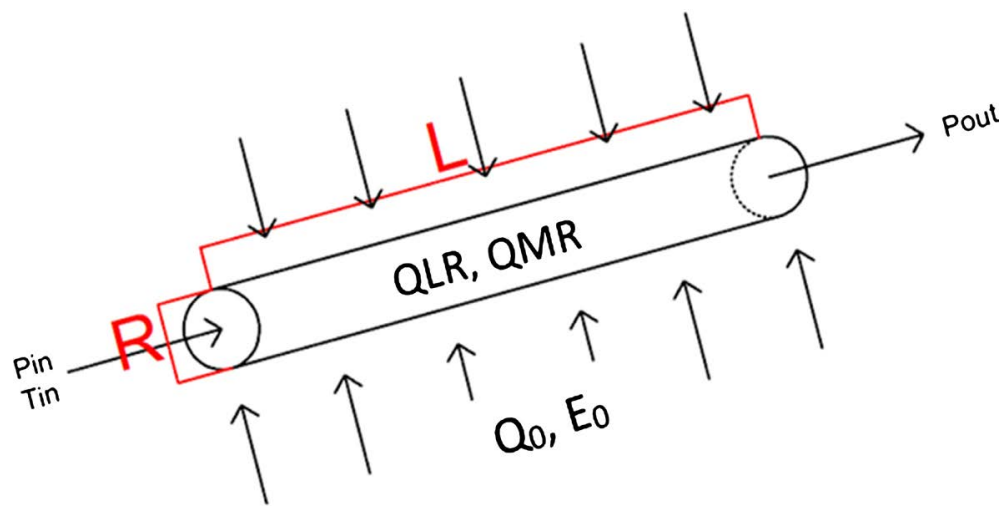

Figure 1. Simple representation of the cylindrical duct. 


$$
\text { @ } r=0, \tau_{r z}=0, \forall z, \forall t>0
$$

Similarly, Equation (3) turns out to be

$$
\rho c_{p}\left(\frac{\partial T}{\partial t}+v_{z} \frac{\partial T}{\partial z}\right)=k\left[\frac{1}{r} \frac{\partial}{\partial r}\left(r \frac{\partial T}{\partial r}\right)+\frac{\partial^{2} T}{\partial z^{2}}\right]+Q
$$

With the following initial and boundary conditions.

As initial condition, the temperature of the fluid is assumed to be uniform

$$
\text { @ } t=0, T=T_{0}, \forall r, z
$$

As boundary conditions for the radial direction we consider the symmetry respect the $z$-axis and the heat exchange at the wall by external convection.

$$
\begin{gathered}
@ r=0, \frac{\partial T}{\partial r}=0, \forall z, t \\
@ r=R, k \frac{\partial T}{\partial r}=-h c *\left(T-T_{a i r}\right), \forall z, t
\end{gathered}
$$

With initial and boundary conditions for the radial direction, so defined, the system results to be axial-symmetric.

For the $z$ direction uniform temperature in the inlet and Danckwerts's condition in the outlet section are imposed

$$
\begin{aligned}
& \text { @ } \mathrm{z}=0, T=T_{i n}, \forall r, t \\
& \text { @ } \mathrm{z}=L, \frac{\partial T}{\partial \mathrm{z}}=0, \forall r, t
\end{aligned}
$$

Initial temperature $T_{0}$ and input temperature $T_{\text {in }}$ are both equal to environmental temperature $T_{\text {air }}$.

Heat generation due to microwaves can be modeled as the $Q L R$ predicted by Lambert's law or as the $Q M R$ provided by the Poynting theorem after the resolution of Maxwell equations.

Lambert's law forecasts an exponential decay inside the sample, Romano et al. (2005)

$$
Q L R=2 \alpha \cdot\left(\frac{R}{r}\right) \cdot Q_{1} \cdot\left[\mathrm{e}^{-2 \alpha(R-r)}+\mathrm{e}^{-2 \alpha(R+r)}\right]
$$

where

$$
\begin{gathered}
Q_{1}=\frac{Q_{0}}{2 \pi R(R+L)} \\
\alpha=\frac{2 \pi}{\lambda_{0}} \sqrt{\frac{\varepsilon^{\prime}\left\{\left[1+\left(\frac{\varepsilon^{\prime \prime}}{\varepsilon^{\prime}}\right)^{2}\right]^{\frac{1}{2}}-1\right\}}{2}}
\end{gathered}
$$

$\frac{\varepsilon^{\prime \prime}}{\varepsilon^{\prime}}$ is the loss tangent, known as $\tan \delta$.

The attenuation factor $\alpha$ for each fluid has been considered as a temperature function, calculated by interpolation starting from graphic relationships for di- 
electric constant and loss tangent versus temperature in a range $10^{\circ} \mathrm{C}-90^{\circ} \mathrm{C}[16]$.

Maxwell equations are a system of four vectory equations [17] [18]:

$$
\begin{gathered}
\nabla \times \boldsymbol{E}=-\frac{\partial \boldsymbol{B}}{\partial t} \text { Faraday's law } \\
\nabla \times \boldsymbol{H}=\frac{\partial \boldsymbol{D}}{\partial t}+\boldsymbol{J}+\sigma \boldsymbol{E} \text { Ampère-Maxwell law } \\
\nabla \cdot \boldsymbol{D}=\rho \text { Gauss's law for electric field } \\
\nabla \cdot \boldsymbol{B}=0 \text { Gauss's law for magnetic field }
\end{gathered}
$$

Keeping in mind also the following constitutive equations:

$$
\begin{aligned}
\boldsymbol{D} & =\varepsilon \boldsymbol{E} \\
\boldsymbol{B} & =\mu \boldsymbol{H}
\end{aligned}
$$

Making the rotor of first and second member of (8)

$$
\nabla \times(\nabla \times \boldsymbol{E})=\nabla \times\left(-\mu \frac{\partial \boldsymbol{H}}{\partial t}\right)
$$

Hence

$$
\nabla(\nabla \cdot \boldsymbol{E})-\nabla^{2} \boldsymbol{E}=-\mu \frac{\partial}{\partial t}(\nabla \times \boldsymbol{H})
$$

Using Equations (9) and (10)

$$
\begin{gathered}
\nabla \frac{\rho}{\varepsilon}-\nabla^{2} \boldsymbol{E}=-\mu \varepsilon \frac{\partial^{2} \boldsymbol{E}}{\partial t^{2}}-\mu \frac{\partial \boldsymbol{J}}{\partial t}-\mu \sigma \frac{\partial \boldsymbol{E}}{\partial t} \\
\nabla^{2} \boldsymbol{E}=\frac{1}{\varepsilon} \nabla \rho+\mu \sigma \frac{\partial \boldsymbol{E}}{\partial t}+\mu \varepsilon \frac{\partial^{2} \boldsymbol{E}}{\partial t^{2}}
\end{gathered}
$$

For source-free region ( $\rho=0$ ) and media with negligible electrical conductivity $(\sigma \approx 0)$ it becomes:

$$
\nabla^{2} \boldsymbol{E}-\frac{1}{c^{2}} \frac{\partial^{2} \boldsymbol{E}}{\partial t^{2}}=0
$$

that is the wave equation in which $c=\sqrt{\frac{1}{\mu \varepsilon}}$ is the speed of light $(299,792,458 \mathrm{~m} / \mathrm{s})$.

It can be noticed that solution of the latter equation, that is second order respect to time, is equivalent to solution of the original system made by two first order equations. Passing from time domain to frequency domain:

$$
\nabla^{2} \boldsymbol{E}+k^{2} \boldsymbol{E}=0
$$

where

$$
k=\omega \sqrt{\mu \varepsilon}=\frac{\omega}{c}=\frac{2 \pi f}{c}=\frac{2 \pi}{\lambda}
$$

The equation assumes the following form:

$$
\nabla^{2} \boldsymbol{E}+\omega^{2} \mu \varepsilon \boldsymbol{E}=0
$$

Anyway, once solved for electric field, magnetic field $H$ can be easily found using Equation (9). 
After that electric and magnetic fields have been calculated, one can also determine the volumetric unit power generated by the correspondent wave thanks to the so called Poynting theorem.

Poynting vector $\boldsymbol{S}[=] \frac{\mathrm{W}}{\mathrm{m}^{2}}$ is defined as the vector product $\boldsymbol{E} \times \boldsymbol{H}$ and represents the directional energy flux of an electromagnetic field.

Poynting's theorem is a statement of conservation of energy for the electromagnetic field. In differential form, defining energy density as $e$ :

$$
\begin{gathered}
-\frac{\partial e}{\partial t}=\nabla \cdot \boldsymbol{S}+\boldsymbol{J} \cdot \boldsymbol{E}[=] \frac{\mathrm{W}}{\mathrm{m}^{3}} \\
-\frac{\partial e}{\partial t}=\nabla \cdot(\boldsymbol{E} \times \boldsymbol{H})+\boldsymbol{J} \cdot \boldsymbol{E} \\
-\frac{\partial e}{\partial t}=\boldsymbol{H} \cdot \nabla \times \boldsymbol{E}-\boldsymbol{E} \cdot \nabla \times \boldsymbol{H}+\boldsymbol{J} \cdot \boldsymbol{E}
\end{gathered}
$$

Using (8), (9), (10) and (11)

$$
\begin{gathered}
\frac{\partial e}{\partial t}=\mu \boldsymbol{H} \frac{\partial \boldsymbol{H}}{\partial t}+\varepsilon \boldsymbol{E} \frac{\partial \boldsymbol{E}}{\partial t} \\
\frac{\partial e}{\partial t}=\frac{1}{2} \frac{\partial}{\partial t}\left(\mu|\boldsymbol{H}|^{2}+\varepsilon|\boldsymbol{E}|^{2}\right)
\end{gathered}
$$

In dielectric materials, the magnetic permeability is usually small and the first term of the equation second side can be neglected; so, shifting to the frequency domain, considering that is $\varepsilon=\varepsilon^{\prime}-j \varepsilon^{\prime \prime}$ and taking only the real component:

$$
\frac{\partial e}{\partial t}=P=\frac{1}{2} \omega \varepsilon_{0} \varepsilon^{\prime \prime}|\boldsymbol{E}|^{2}
$$

\section{Materials and Methods}

To solve the previous partial differential equations, a Finite Elements Method (FEM) [19] has been used. To practically implement this solution, COMSOL Multiphysics ${ }^{\circledR} 5.3$ has been utilized and in Figure 2 a possible mesh of the system is

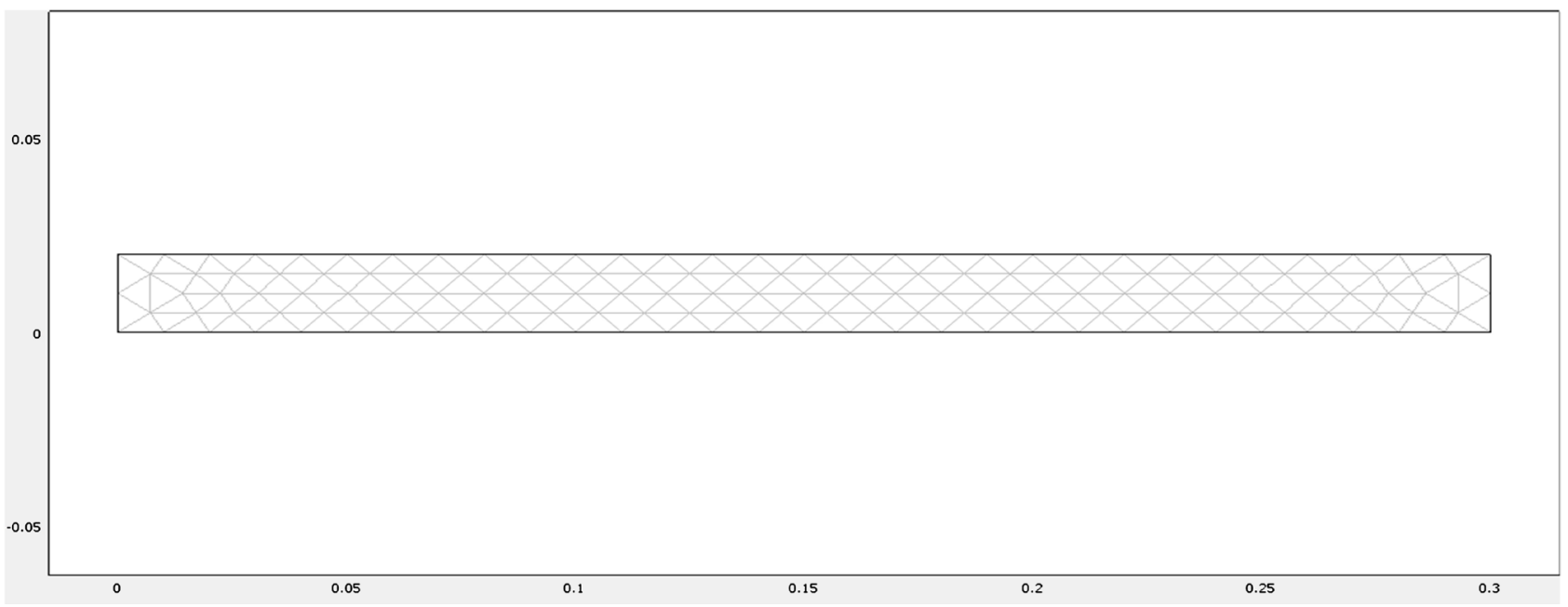

Figure 2. Generic mesh. 
shown.

Three fluid foods have been considered: skim milk, with a Newtonian behavior and constant viscosity, apple sauce and tomato sauce as non Newtonian fluids, modeled with a power law having different fluid consistency coefficient and flow behavior index [20], as reported in Table 1 together with the other physical and rheological properties.

As $\varepsilon^{\prime}$ and $\varepsilon^{\prime \prime}$ are temperature functions, average values have been obtained by integrating in the entire domain and in the time (range $0-100 \mathrm{~s}$ ). They are fundamental for microwave heating, because they determine respectively the energy absorbed and the fraction converted in heat power.

\section{Results}

In the next pages, velocity and temperature profiles and generation term modeled with both Lambert's law and Poynting theorem are reported.

In all cases, the average velocity is the same $(4 \mathrm{~mm} / \mathrm{s})$. To practically realize that, as the three fluid present different rheological properties, different pressure drops, reported in Table 2, have been calculated from integration of the momentum flux distribution for the flow through a circular tube for Newtonian and non-Newtonian fluids.

To ensure a comparability between the two heat generation terms, a proper value of electric field has been chosen as boundary condition in wave equation so that the volume integral over all the domain is the same:

$$
\int_{V} Q L R \mathrm{~d} V=\int_{V} Q M R \mathrm{~d} V
$$

In this way, the total heat supplied in the different cases is the same. Values of total power $P$ for all cases and correspondent values of electric field in case of Poynting generationare reported in Table 3.

Table 1. Physical, dielectric and transport properties.

\begin{tabular}{cccc}
\hline & Skim milk & Apple sauce & Tomato sauce \\
\hline Density, $\rho\left[\mathrm{kg} \mathrm{m}^{-3}\right]$ & 1047.7 & 1104.9 & 1036.9 \\
Specific heat, $c_{p}\left[\mathrm{~J} \mathrm{~kg}^{-1} \mathrm{~K}^{-1}\right]$ & 3943.7 & 3703.3 & 4000.0 \\
Thermal conductivity, $k\left[\mathrm{~W} \mathrm{~m}^{-1} \mathrm{~K}^{-1}\right]$ & 0.5678 & 0.5350 & 0.5774 \\
Viscosity, $\mu\left[\mathrm{Kg} \mathrm{m}^{-1} \mathrm{~s}^{-1}\right]$ & 0.0059 & 32.734 & 3.9124 \\
Fluid consistency coefficient & & 0.197 & 0.097 \\
Flow behavior index & & 68.97 & 74.27 \\
Dielectric constant, $\varepsilon^{\prime}$ & 66.31 & 46.42 & 5.30 \\
Dielectric loss, $\varepsilon^{\prime \prime}$ & 13.26 & &
\end{tabular}

Table 2. Pressure drops applied in the three cases.

\begin{tabular}{cccc}
\hline & Skim milk & Apple sauce & Tomato sauce \\
\hline Pressure drop $[\mathrm{Pa}]$ & 0.1425 & 1080 & 129 \\
\hline
\end{tabular}


All the results showed above are obtained for the same fixed time of 100 seconds. It can be seen that skim milk, that is a Newtonian fluid, shows a parabolic shape (Figure 3), while the other two fluids present a flatter profile. In particular tomato sauce (Figure 5) is flatter than apple sauce (Figure 4), having a lower flow behavior index value $\mathrm{n}$.

The three fluids show a very similar temperature distribution even though apple sauce values are smaller (Figure 6, Figure 7). Temperature profiles can be explained observing the generation terms (Figure 8). Barringer's result of

Table 3. Power absorbed and electric field applied to the three fluids.

\begin{tabular}{cccc}
\hline & Skim milk & Apple sauce & Tomato sauce \\
\hline$P[\mathrm{~W}]$ & 640 & 210 & 750 \\
$E[\mathrm{~V} / \mathrm{m}]$ & 1060 & 1950 & 850 \\
\hline
\end{tabular}
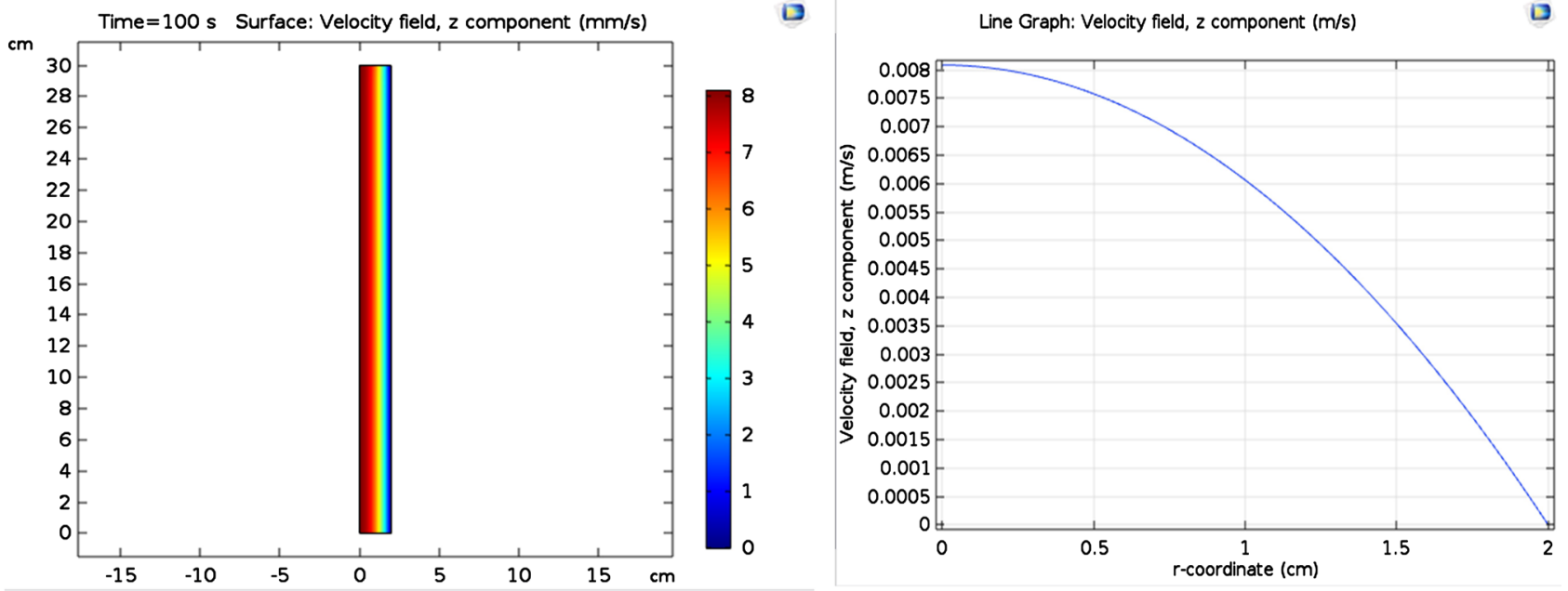

Figure 3. Velocity profile for skim milk.

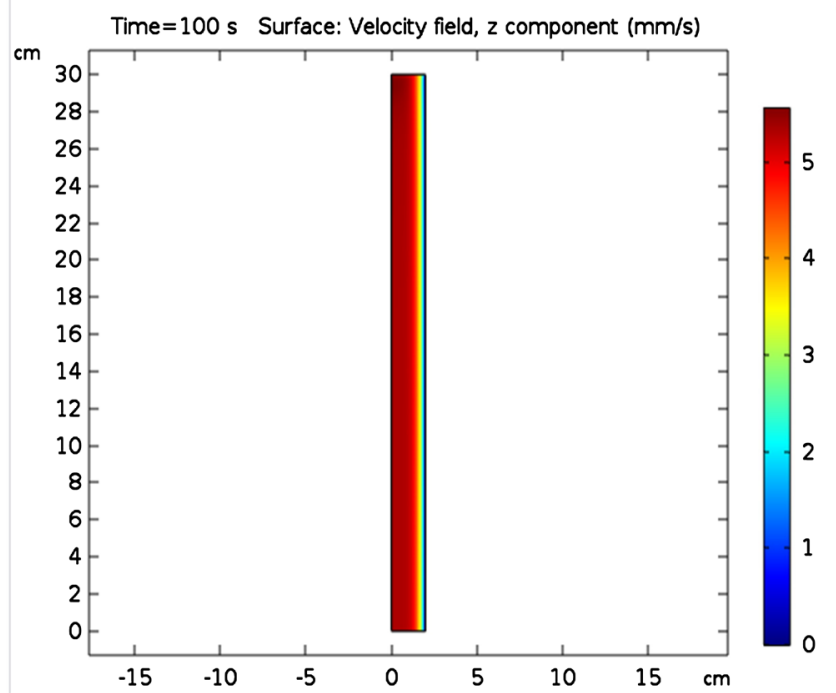

$\square$

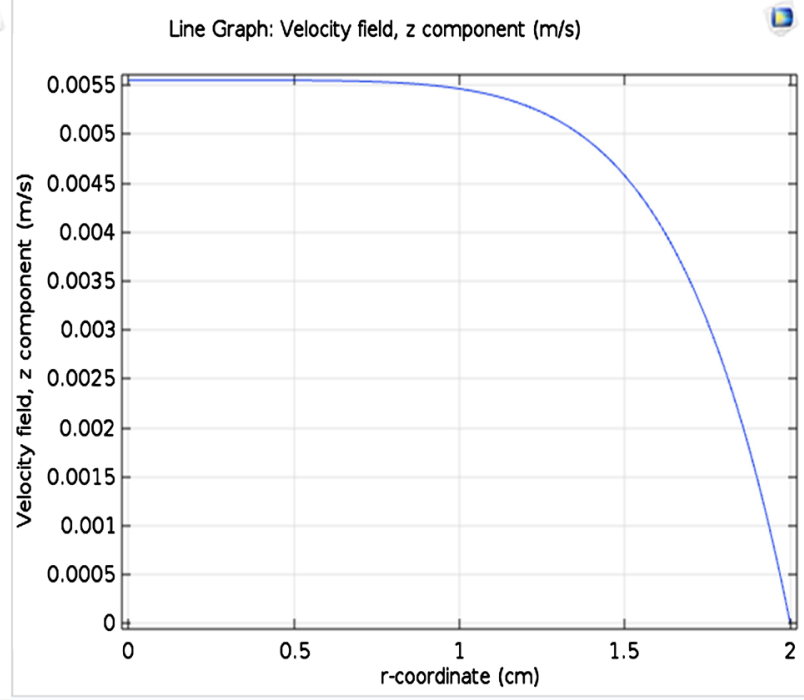

Figure 4. Velocity profile for apple sauce. 

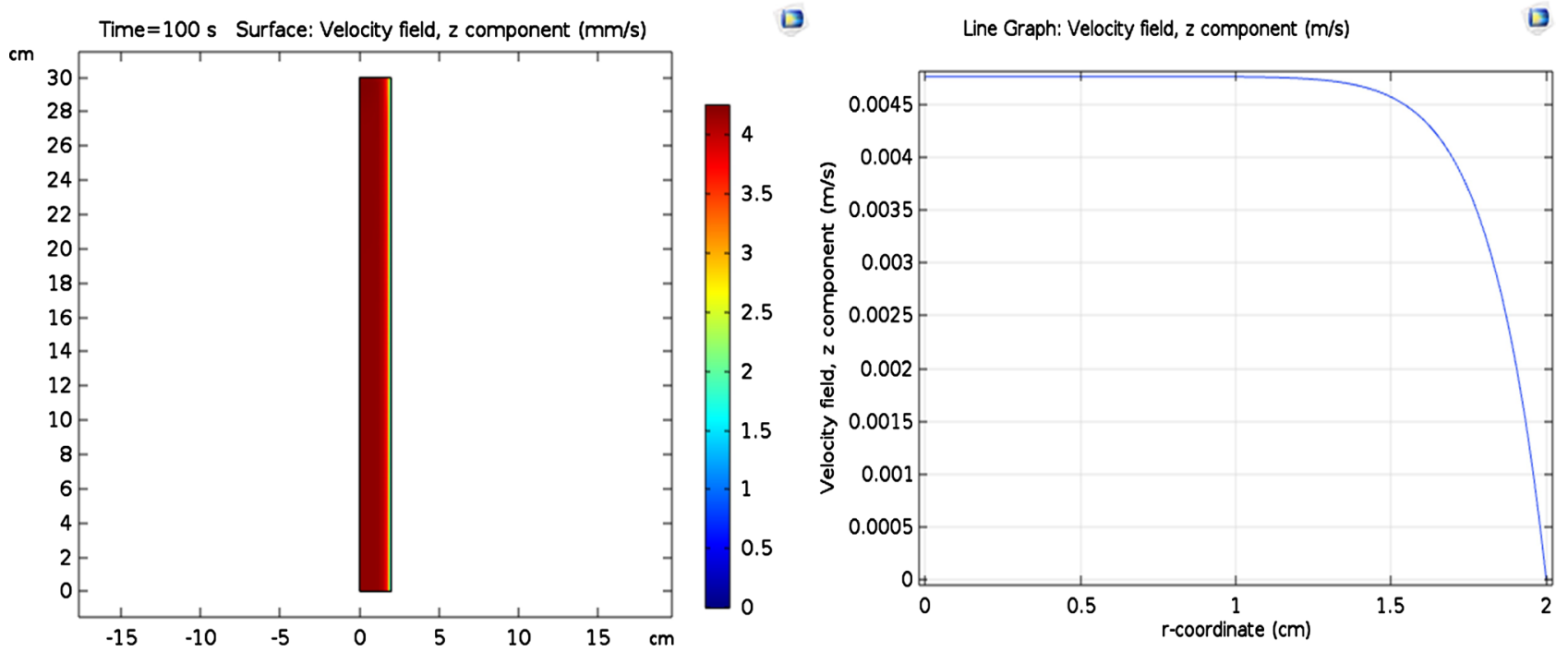

Figure 5. Velocity profile for tomato sauce.
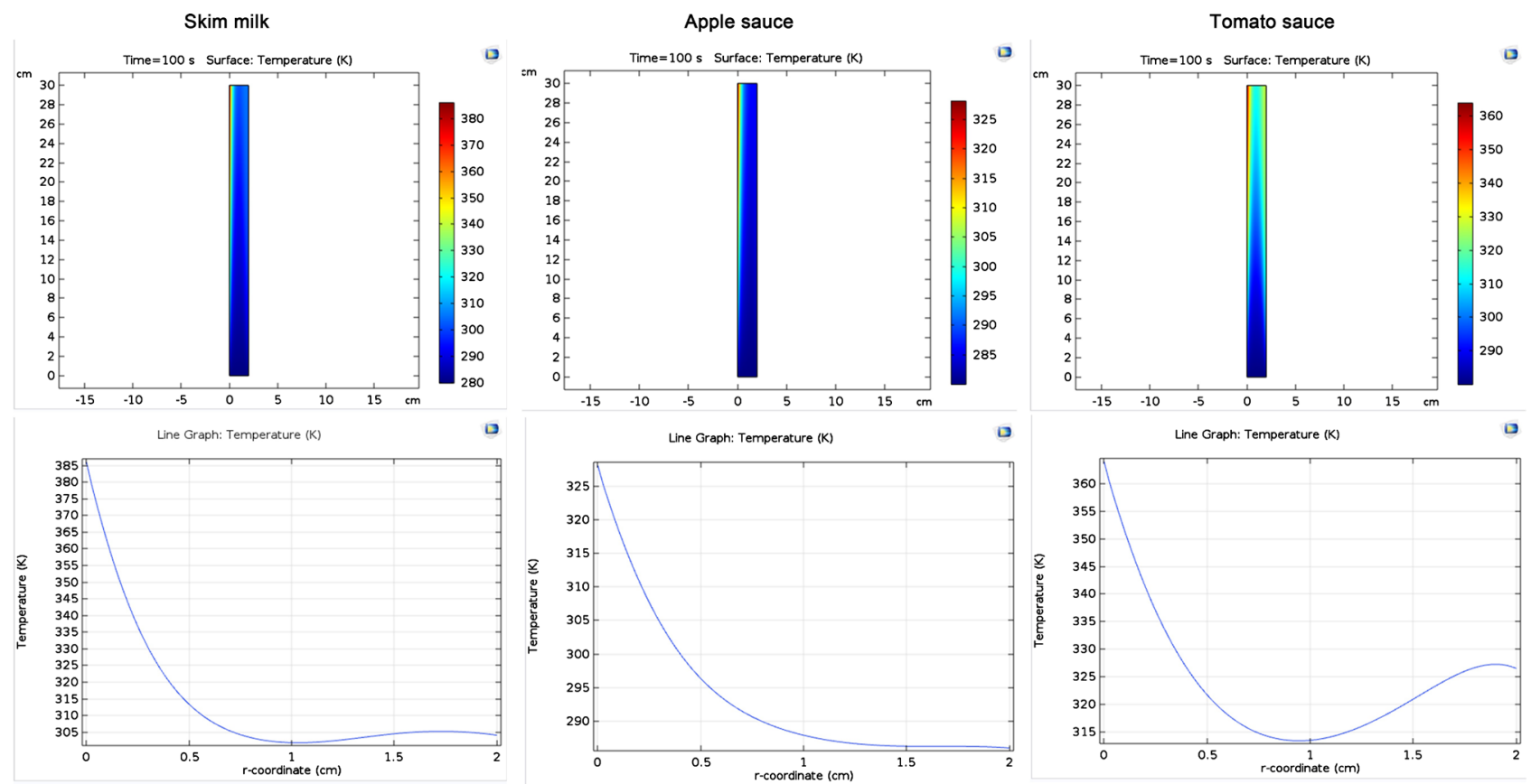

Figure 6. Temperature profile with Lambert generation term.

underestimation of heating in case of Lambert's law use is confirmed also in this case with a different geometry.

Actually, in order to better understand the phenomenon, it has been considered that the fluid elements of each of the three liquids have different velocity profiles along the radial direction (Figures 3-5) and so different residence times depending to the radius. For the fluid elements having higher velocities, near the tube axis, the residence times are lower; for those near the wall, the velocities are lower and so the residence times are longer. In skim milk this difference is more important, whereas in the other two cases the profile is quite flat, especially in 
the case of tomato sauce. Anyway, outlet section $(z=30 \mathrm{~cm})$ has been fixed and temperature profile evaluated for each position based on the real residence time are overlapped in Figure 9.
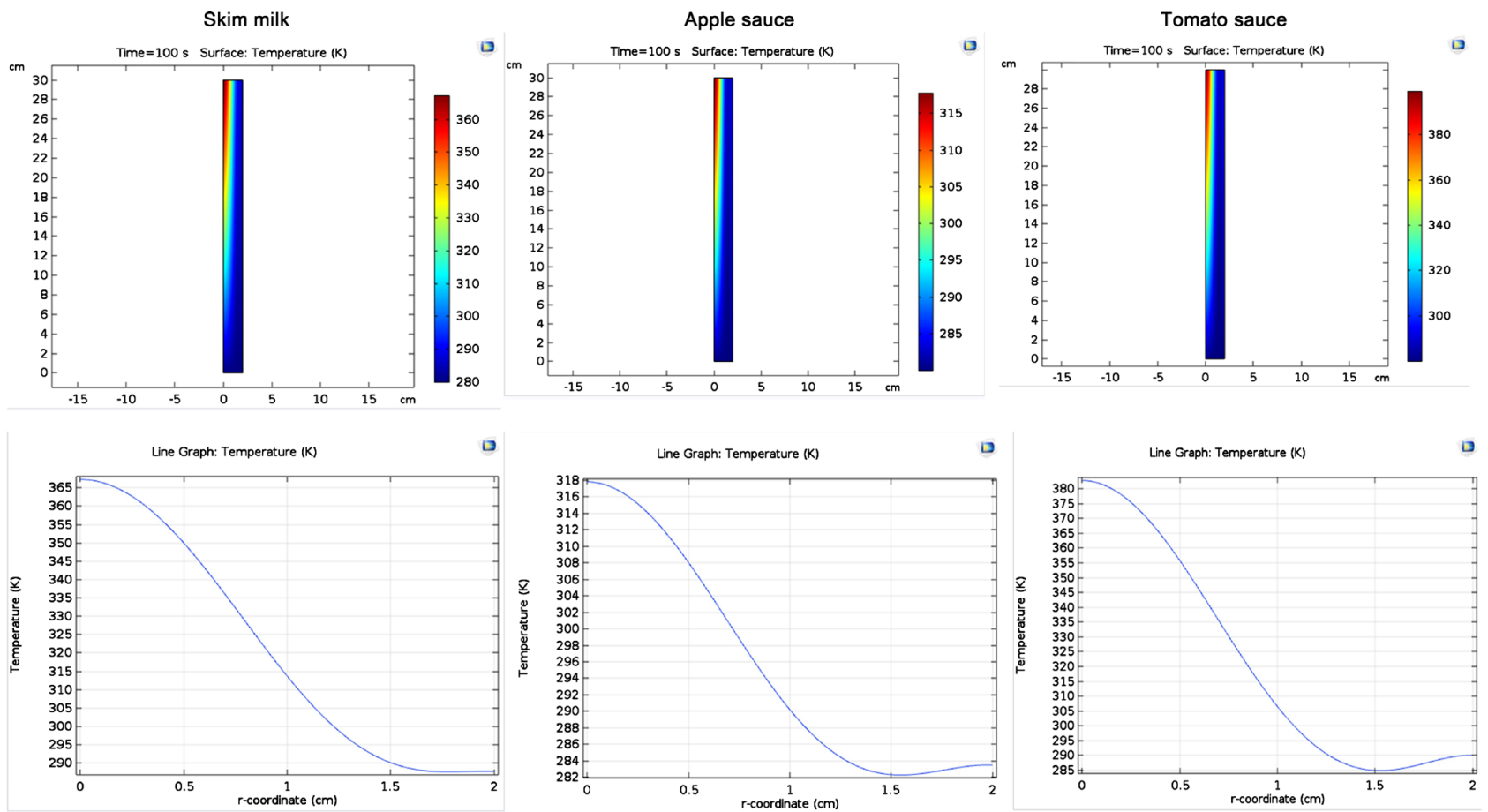

Figure 7. Temperature profiles with Poynting generation term.
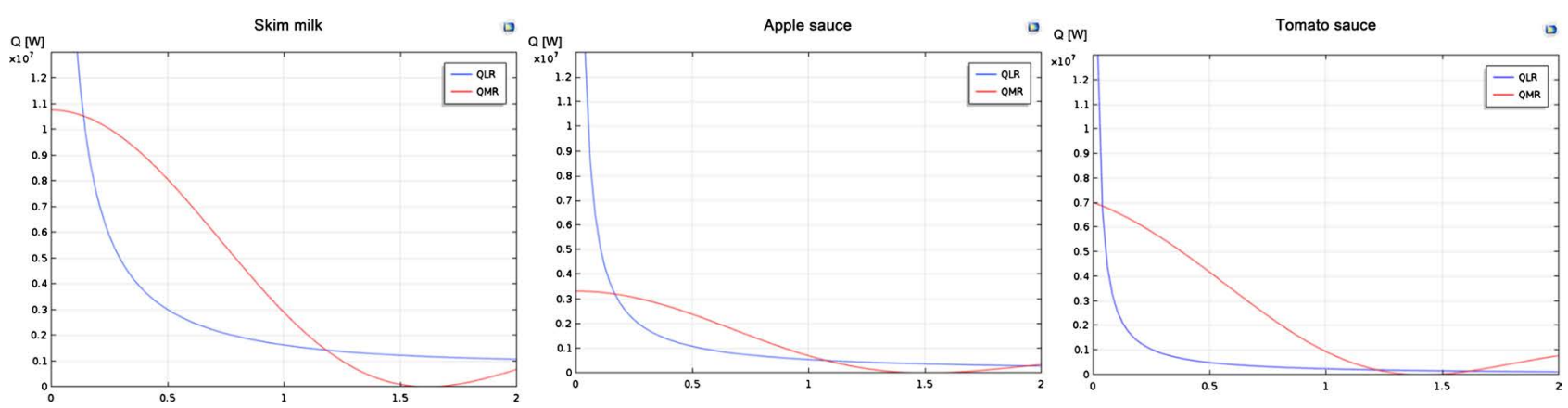

Figure 8. Comparison between $Q L R$ and $Q M R$ for the three liquid foods.

Skim milk

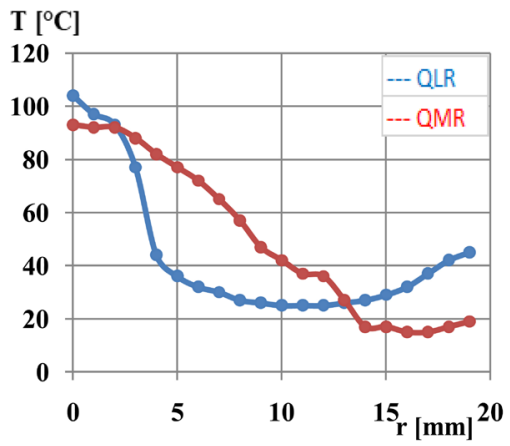

Apple sauce

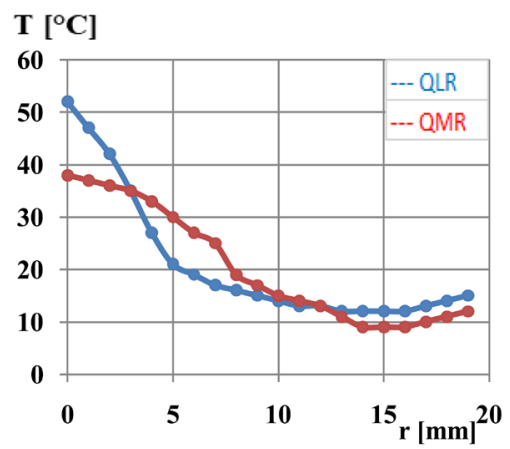

Tomato sauce

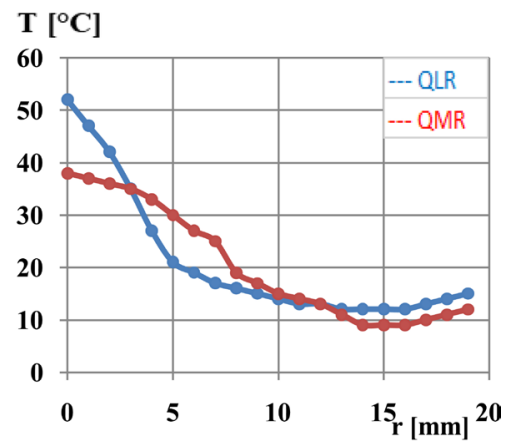

Figure 9. Overlapping of temperature profiles predicted by Lambert's law and Maxwell equations solution. 
It is evident that in the three cases the trend is always the same: in the first part near the axis in which Lambert's laws overestimates Maxwell results in term of temperature. This is due to Lambert's generation term that goes to infinite for $r=0$ that in turn is due to the pre exponential factor $R / r$. In the larger middle part, Lambert's law curve is instead always under Maxwell predictions, save to slightly raise in the last part near the wall.

In both cases temperature increases near the wall because of the longer residence times, but in Lambert's law case the effect is more evident. After all, apart few points, a good agreement between the two models has been found.

\section{Conclusions}

In conclusion, it can be stated that Lambert's law allows to estimate power generated during a microwave heating process without solving for electric field and so in a relatively simply way, but it presents some problems like the singularity of infinite power for $r=0$ and an appreciable underestimation of the real power and so of the temperature distribution. Moreover as it suppose an exponential decay, it can't forecast the oscillations of the electric field and so of Poynting generation.

On the other hand, solution of Maxwell equations allows to exactly know the real electric field and then, thanks to Poynting theorem, the actual generated power, although it is obviously more expensive in terms of time and resource of calculations.

\section{Conflicts of Interest}

The authors declare no conflicts of interest regarding the publication of this paper.

\section{References}

[1] Mermelstein, N.H. (1997) How Food Technology Covered Microwaves over the Years. Food Technology, 51, 82-84.

[2] Ayappa, K.G., Davis, H.T., Davis, E.A. and Gordon J, (1991) Analysis of Microwave Heating of Materials with Temperature Dependent Properties. AIChE Journal, 37, 313-322. https://doi.org/10.1002/aic.690370302

[3] Chatterjee, A., Basak, T. and Ayappa, K.G. (1998) Analysis of Microwave Sintering of Ceramics AIChE Journal, 44, 2302-2311. https://doi.org/10.1002/aic.690441019

[4] O'Brien, K.T. and Mekkaoui, A.M. (1993) Numerical Simulation of the Thermal Fields Occurring in the Treatment of Malignant Tumors by Local Hyperthermia. Journal of Biomechanical Engineering, 115, 247-253. https://doi.org/10.1115/1.2895482

[5] Paulsen, K.D., Lynch, D.R. and Strohbehn, J.W. (1998) Three-Dimensional Finite, Boundary, and Hybrid Element Solutions of the Maxwell Equations for Lossy Dielectric Media. IEEE Transactions on Microwave Theory and Techniques, 36, 682-693.

[6] Ayappa, K.G., Davis, H.T., Davis, E.A. and Gordon, J. (1992) Two Dimensional Finite Elements Analysis of Microwave Heating. AIChE Journal, 38, 1577-1592. 
https://doi.org/10.1002/aic.690381009

[7] Oliveira, M.E.C. and Franca, A.S. (2002) Microwave Heating of Foodstuffs. Journal of Food Engineering, 53, 347-359. https://doi.org/10.1016/S0260-8774(01)00176-5

[8] Lin, Y.E., Anantheswaran, R.C. and Puri, V.M. (1995) Finite Element Analysis of Microwave Heating of Solid Foods. Journal of Food Engineering, 25, 85-112. https://doi.org/10.1016/0260-8774(94)00008-W

[9] Zhou, L., Puri, V.M., Anantheswaran, R.C. and Yeh, G. (1995) Finite Element Modeling of Heat and Mass Transfer in Food Materials during Microwave Heating-Model Development and Validation. Journal of Food Engineering, 25, 509-529. https://doi.org/10.1016/0260-8774(94)00032-5

[10] Romano, V., Marra, F. and Tammaro, U. (2005) Modelling of Microwave Heating of Foodstuff: Study on the Influence of Sample Dimensions with a FEM Approach. Journal of Food Engineering, 71, 233-241. https://doi.org/10.1016/j.jfoodeng.2004.11.036

[11] Ratanadecho, P., Aoki, K. and Akahori, M. (2002) A Numerical and Experimental Investigation of the Modeling of Microwave Heating for Liquid Layers Using a Rectangular Wave Guide (Effects of Natural Convection and Dielectric Properties). Applied Mathematical Modelling, 26, 449-472. https://doi.org/10.1016/S0307-904X(01)00046-4

[12] Zhang, Q., Jackson, T.H. and Ungan, A. (2000) Numerical Modeling of Microwave Induced Natural Convection. Journal of Heat and Mass Transfer, 43, 2141-2154. https://doi.org/10.1016/S0017-9310(99)00281-1

[13] Romano, V. and Apicella, R. (2015) Microwave Heating of Liquid Foods. Engineering, 7, 297-306. https://doi.org/10.4236/eng.2015.76026

[14] Barringer, S.A., Davis, E.A., Gordon, J., Ayappa, K.G. and Davis, H.T. (1995) Microwave-Heating Temperature Profiles for Thin Slabs Compared to Maxwell and Lambert Law Predictions. Journal of Food Science, 60, 1137-1142.

[15] Bird, R.B., Stewart, W.E. and Lightfoot E.N. (2002) Transport Phenomena.

[16] Zhu, J., Kuznetsov, A.V. and Sandeep, K.P. (2007) Mathematical Modeling of Continuous Flow Microwave Heating of Liquids (Effects of Dielectric Properties and Design Parameters). International Journal of Thermal Sciences, 46, 328-341. https://doi.org/10.1016/j.ijthermalsci.2006.06.005

[17] Balanis, A.C. (1989) Advanced Engineering Electromagnetics.

[18] Collin, R.E. (1992) Foundations for Microwave Engineering.

[19] Zienkiewicz, O.C. (2000) The Finite Element Method.

[20] Steffe, J.F. (1992) Rheological Methods in Food Process Engineering. 


$\begin{array}{lll}\text { Symbol } & \text { Nomenclature } & \text { Units } \\ L & \text { tube length } & {[\mathrm{m}]} \\ R & \text { tube radius } & {[\mathrm{m}]} \\ Z & \text { axial direction } & {[\mathrm{m}]} \\ r & \text { radial direction } & {[\mathrm{m}]} \\ V & \text { fluid velocity } & {\left[\mathrm{ms}^{-1}\right]} \\ Q_{0} & \text { incident microwave power at the sample surface } & {\left[\mathrm{Wm}^{-2}\right]} \\ f & \text { microwave frequency } & {\left[\mathrm{s}^{-1}\right]} \\ \lambda_{0} & \text { microwave wavelength } & {\left[\mathrm{m}^{-1}\right.} \\ c & \text { speed of light constant } & {\left[\mathrm{ms}^{-1}\right]} \\ a & \text { attenuation factor } & {\left[\mathrm{m}^{-1}\right]} \\ \varepsilon^{\prime} & \text { dielectric constant } & \\ \varepsilon^{\prime \prime} & \text { dielectric loss } & \\ \delta & \text { penetration depth } & {\left[\mathrm{m}^{2}\right]} \\ T & \text { temperature } & {[\mathrm{K}]} \\ t & \text { time } & {[\mathrm{s}]} \\ P & \text { pressure } & {\left[\mathrm{Pa}^{-1}\right.} \\ g & \text { gravitational acceleration } & {\left[\mathrm{ms}^{-2}\right]} \\ Q L R & \text { heat generation due to microwave, Lambert's law } & {\left[\mathrm{Wm}^{-3}\right]} \\ Q M R & \text { heat generation due to microwave, Maxwell's equations }\left[\mathrm{Wm}^{-3}\right] \\ h_{c} & \text { convective heat transfer coefficient } & {\left[\mathrm{Wm}^{-2} \mathrm{~K}^{-1}\right]} \\ E & \text { electric field vector } & {\left[\mathrm{Vm}^{-1}\right]} \\ H & \text { magnetic field vector } & {\left[\mathrm{Am}^{-1}\right]}\end{array}$

